MATHEMATICS OF COMPUTATION

Volume 69, Number 231, Pages 1053-1070

S 0025-5718(99)01161-8

Article electronically published on August 25, 1999

\title{
ALMOST PERIODIC FACTORIZATION OF CERTAIN BLOCK TRIANGULAR MATRIX FUNCTIONS
}

\author{
ILYA M. SPITKOVSKY AND DARRYL YONG
}

AbStract. Let

$$
G(x)=\left[\begin{array}{cc}
e^{i \lambda x} I_{m} & 0 \\
c_{-1} e^{-i \nu x}+c_{0}+c_{1} e^{i \alpha x} & e^{-i \lambda x} I_{m}
\end{array}\right],
$$

where $c_{j} \in \mathbb{C}^{m \times m}, \alpha, \nu>0$ and $\alpha+\nu=\lambda$. For rational $\alpha / \nu$ such matrices $G$ are periodic, and their Wiener-Hopf factorization with respect to the real line $\mathbb{R}$ always exists and can be constructed explicitly. For irrational $\alpha / \nu$, a certain modification (called an almost periodic factorization) can be considered instead. The case of invertible $c_{0}$ and commuting $c_{1} c_{0}^{-1}, c_{-1} c_{0}^{-1}$ was disposed of earlier-it was discovered that an almost periodic factorization of such matrices $G$ does not always exist, and a necessary and sufficient condition for its existence was found.

This paper is devoted mostly to the situation when $c_{0}$ is not invertible but the $c_{j}$ commute pairwise $(j=0, \pm 1)$. The complete description is obtained when $m \leq 3$; for an arbitrary $m$, certain conditions are imposed on the Jordan structure of $c_{j}$. Difficulties arising for $m=4$ are explained, and a classification of both solved and unsolved cases is given.

The main result of the paper (existence criterion) is theoretical; however, a significant part of its proof is a constructive factorization of $G$ in numerous particular cases. These factorizations were obtained using Maple; the code is available from the authors upon request.

\section{INTRODUCTION}

Let $A P$ be the Bohr algebra of almost periodic functions, that is, the smallest $C^{*}$-algebra of $L^{\infty}(\mathbb{R})$ containing all the functions $e_{\lambda}(x)=e^{i \lambda x}, \lambda \in \mathbb{R}$. It is well known (the standard references for these and other properties of $A P$ are [3 11, 12]) that for every $f \in A P$,

1. there exists the Bohr mean value

$$
\mathbf{M}(f)=\lim _{T \rightarrow \infty} \frac{1}{2 T} \int_{-T}^{T} f(x) d x
$$

and

2. the Fourier coefficients $\widehat{f}(\lambda) \stackrel{\text { def }}{=} \mathbf{M}\left(f e_{-\lambda}\right)$ are different from zero for at most countably many values of $\lambda \in \mathbb{R}$.

Received by the editor March 12, 1997 and, in revised form, September 18, 1998.

1991 Mathematics Subject Classification. Primary 47A68, 47-04, 42A75.

Key words and phrases. Almost periodic matrix functions, factorization, explicit computation.

The first author's research was partially supported by NSF Grant DMS-9800704.

The second author's research was started during a Research Experience for Undergraduates sponsored by the NSF at the College of William and Mary during the summer of 1995 . 
The set $\Omega(f)=\{\mu: \widehat{f}(\mu) \neq 0\}$ is called the Fourier spectrum of $f$, and

$$
\sum_{\mu \in \Omega(f)} \widehat{f}(\mu) e_{\mu}
$$

is its (formal) Fourier series.

We say that $f \in A P_{W}$ if the Fourier series (1.1) converges absolutely:

$$
\sum_{\mu \in \Omega(f)}|\widehat{f}(\mu)|<\infty
$$

Finally, let

$$
A P^{ \pm}=\left\{f \in A P: \Omega(f) \subset \mathbb{R}_{ \pm}\right\} \quad \text { and } \quad A P_{W}^{ \pm}=A P^{ \pm} \cap A P_{W} .
$$

Here, as usual, $\mathbb{R}_{ \pm}=\{x \in \mathbb{R}: \pm x \geq 0\}$.

For matrix functions $f$, conditions $f \in A P, A P^{ \pm}, A P_{W}$, etc. are understood entrywise, and $\mathbf{M}(f), \widehat{f}(\mu), \Omega(f)$ are defined by exactly the same formulas as for scalar functions.

Following 6, we introduce an $A P$ factorization of an $n \times n$ matrix function $G$ as its representation in the form

$$
G=G_{+} \Lambda G_{-},
$$

where $\Lambda(x)=\operatorname{diag}\left[e_{\lambda_{1}}, \ldots, e_{\lambda_{n}}\right]$,

$$
G_{+}^{ \pm 1} \in A P^{+}, G_{-}^{ \pm 1} \in A P^{-},
$$

and $\lambda_{1}, \ldots, \lambda_{n} \in \mathbb{R}$. We say that (1.2) is an $A P_{W}$ factorization of $G$ if conditions (1.3) are replaced by the (more restrictive) conditions $G_{+}^{ \pm 1} \in A P_{W}^{+}, G_{-}^{ \pm 1} \in A P_{W}^{-}$.

If $G$ is $A P$ factorable, the numbers $\lambda_{1}, \ldots, \lambda_{n}$ are defined uniquely; they are called the partial $A P$ indices (of $G$ ). Of course, for an $A P\left(A P_{W}\right)$ factorization (1.2) to exist it is necessary that $G^{ \pm 1} \in A P$ (respectively, $A P_{W}$ ). However, this necessary condition is not sufficient and, except for the case of periodic matrix functions $G$ (in which an $A P$ factorization by a simple change of variable reduces to the usual Wiener-Hopf factorization), the theory of $A P$ factorization is "under construction". Its connections with integral equations, completion problems, and signal processing are discussed in [6, 7], 17, 15, 1], and [14 respectively. Explicit formulas for the factors in (1.2) for certain special types of $G$ are obtained in [6, 9, 8 . Most of them refer to matrices $G$ of the following block triangular form:

$$
G_{f}=\left[\begin{array}{cc}
e_{\lambda} I_{m} & 0 \\
f & e_{-\lambda} I_{m}
\end{array}\right]
$$

(so that $n=2 m$ ) arising in the treatment of convolution type equations on finite intervals of length $\lambda$.

In particular, the following two statements were established in $[6]$.

Lemma 1.1. Let $f$ be an $A P_{W}$ matrix function, and let

$$
f_{0}=\sum_{\mu \in \Omega(f) \cap(-\lambda, \lambda)} \widehat{f}(\mu) e_{\mu} .
$$

Then the matrices $G_{f}$ and $G_{f_{0}}$ are $A P\left(A P_{W}\right)$ factorable only simultaneously, and their partial AP indices coincide.

Due to Lemma [1.1, for any $f \in A P_{W}$ in (1.4) we may suppose without loss of generality that $\Omega(f) \subset(-\lambda, \lambda)$. 
Theorem 1.2. Let $\Omega(f) \cap(-\lambda, \lambda)$ consist of at most two points, say $\mu$ and $\sigma$. Then $G_{f}$ is $A P_{W}$ factorable. Its partial $A P$ indices all equal zero if and only if $\mu \sigma=0$, $\widehat{f}(0)$ is invertible, or $\mu \sigma<0, \frac{\lambda}{\mu-\sigma} \in \mathbb{Z}$ and both $\widehat{f}(\mu), \widehat{f}(\sigma)$ are invertible.

The next logical step is to consider a trinomial $f$ with $\Omega(f) \subset(-\lambda, \lambda)$. However, with no additional restrictions on the location of $\Omega(f)$ this remains an open problem. In this paper, we concentrate on the case $\Omega(f)=\{-\nu, 0, \alpha\}$, that is,

$$
f=c_{-1} e_{-\nu}+c_{0}+c_{1} e_{\alpha},
$$

where $\alpha, \nu>0$ and $\alpha+\nu=\lambda$.

If $\beta=\frac{\nu}{\alpha}$ is rational, then the matrix $G_{f}$ is periodic, and its $A P_{W}$ factorization exists and can be easily constructed. Thus, we suppose in what follows that $\beta$ is irrational. The next result applies to the case when the matrices $c_{j}$ in (1.5) commute with each other. In this case there exists a similarity $T$ such that

$$
T^{-1} c_{j} T=\operatorname{diag}\left[c_{j 1}, \ldots, c_{j r}\right], \quad c_{j k} \in \mathbb{C}^{l_{k} \times l_{k}}, k=1, \ldots, r ; j=0, \pm 1,
$$

and each diagonal block $c_{j k}$ has a singleton spectrum (see [13, Section 4.4]):

$$
\sigma\left(c_{j k}\right)=\left\{\xi_{j k}\right\} \quad(j=0, \pm 1 ; k=1, \ldots r) .
$$

As in [2, we call $\left\{\xi_{j k}\right\}_{j=-1}^{1}$ the bonded eigenvalue triples of $c_{j}$.

Theorem 1.3. Let $G_{f}$ be of the form (1.4) with $f$ given by (1.5) and commuting coefficients $c_{j}$. Then $G_{f}$ is AP factorable with zero partial AP indices if and only if, for all bonded triples $\left\{\xi_{-1, k}, \xi_{0, k}, \xi_{1, k}\right\}$,

$$
\left|\xi_{1, k}^{\nu} \xi_{-1, k}^{\alpha}\right| \neq\left|\xi_{0, k}\right|^{\lambda} \quad(k=1, \ldots, r)
$$

In this form, Theorem 1.3 was established in [2, Theorem 7.2]; the case of invertible $c_{j}$ was disposed of earlier in [9]. In fact, the result of [9] contains an additional important piece of information: if all $c_{j}$ are invertible and (1.7) fails for at least one value of $k$, then $G_{f}$ does not admit any $A P$ factorization, even if non-zero partial $A P$ indices are allowed. Also, it was shown in [16] that an $A P$ factorization with zero partial $A P$ indices of an $A P_{W}$ matrix function is automatically its $A P_{W}$ factorization. Hence, the following result holds.

Corollary 1.4. Let $G_{f}$ be as in Theorem 1.3 and, in addition, let $c_{0}$ be invertible. Then $G_{f}$ is $A P_{W}$ factorable with zero partial AP indices if condition (1.7) holds, and is not $A P$ factorable otherwise.

Of course, it would now be natural to consider an $A P$ factorization of $G_{f}$ with trinomial $f$, pairwise commuting $c_{j}$, and no restrictions imposed on the invertibility of $c_{0}$ and the values of partial $A P$ indices. We will see, however, that this problem embraces a general setting of a trinomial $f$ with arbitrary (not necessarily commuting) coefficients $c_{j}$ and is therefore too difficult to handle at the present stage of the development. Our paper is a report on several partial results on the $A P$ factorability of matrices (1.4), (1.5) with non-invertible $c_{0}$.

The paper is structured as follows. Section 2 contains an auxiliary result on the factorization of block diagonal matrices. It also describes a procedure which allows us to replace a matrix of the form (1.4), (1.5) with invertible $c_{-1}$ (and no commutativity conditions on $c_{j}$ ) by another matrix of the same type without changing its factorability properties. This procedure is, in fact, a variation of the one introduced in [2] for matrices (1.4) with a finite number (not limited to three) of 
points $\mu_{j}$ in $\Omega(f) \cap(-\lambda, \lambda)$ but pairwise commuting $\widehat{f}\left(\mu_{j}\right)$. As a direct application of this procedure, $A P_{W}$ factorability is established for matrices (1.4), (1.5) with $m=2$, invertible $c_{-1}$ (or $c_{1}$ ) and nilpotent $c_{0} c_{-1}^{-1}$ (respectively, $c_{0} c_{1}^{-1}$ ).

Section 3 contains necessary and sufficient factorability conditions for matrices (1.4), (1.5) with commuting $c_{j}$ under certain additional restrictions on their Jordan structure. This covers, in particular, all matrices of size $m \leq 3$, invertible $c_{1}$ or $c_{-1}$ of size $m \leq 4$, and matrices of arbitrary size, provided that each eigenvalue of at least one of the $c_{j}$ corresponds to one Jordan cell. An application to difference equations is given.

In Section 4 , we concentrate on $4 \times 4$ matrices $c_{j}$. An example is given explaining why this case cannot be covered in general before the $A P$ factorability of matrices (1.4), (1.5) with arbitrary invertible non-commuting $c_{j}$ is understood. All possible cases are classified, and those for which the $A P$ factorability remains unknown are singled out.

Proofs of the results in Sections 3 and 4 are partially theoretical and partially consist in exhausting a large number of cases in which an $A P_{W}$ factorization can be constructed explicitly. These cases are relegated to Section 5 the supplement at the end of this volume, where final formulas are listed. Of course, they can be checked by straightforward calculations. We emphasize, however, that a symbolic manipulation Maple program was used to obtain these formulas, and without it this paper could hardly have been completed.

\section{Auxiliary Results}

Suppose $G$ is a block diagonal $A P$ matrix: $G=\operatorname{diag}\left[G_{1}, G_{2}\right]$. If its diagonal blocks $G_{1}, G_{2}$ are $A P$ factorable, then $G$ itself is $A P$ factorable. Moreover, an $A P$ factorization of $G$ can be obtained by "pasting together" $A P$ factorizations of $G_{1}$ and $G_{2}: G_{1}=G_{+}^{(1)} \Lambda^{(1)} G_{-}^{(1)}, G_{2}=G_{+}^{(2)} \Lambda^{(2)} G_{-}^{(2)}$ imply

$$
G=\operatorname{diag}\left[G_{+}^{(1)}, G_{+}^{(2)}\right] \operatorname{diag}\left[\Lambda^{(1)}, \Lambda^{(2)}\right] \operatorname{diag}\left[G_{-}^{(1)}, G_{-}^{(2)}\right] .
$$

It is natural to ask whether the converse is true. The answer is positive provided that $G \in A P_{W}$ and partial $A P$ indices of $G$ equal zero. Indeed, a matrix $F \in$ $A P_{W}$ admits an $A P$ factorization with zero partial $A P$ indices if and only if the corresponding Toeplitz operator $T_{F}$ is invertible on $L^{2}\left[5\right.$ (see also [7]). Since $T_{G}$ is a direct sum of $T_{G_{1}}$ with $T_{G_{2}}$, the invertibility of $T_{G}$ is equivalent to simultaneous invertibility of $T_{G_{1}}$ and $T_{G_{2}}$.

We are not aware of any equivalent of $A P$ factorability (with non-zero partial $A P$ indices) in operator terms. Probably, the answer to the question is still positive, but we restrict our consideration to a somewhat weaker version.

Lemma 2.1. Let $G=\operatorname{diag}\left[G_{1}, G_{2}\right]$. If $G$ and one of its diagonal blocks $G_{1}, G_{2}$ are AP factorable, then the other diagonal block is also AP factorable.

Proof. Consider first the case when $G_{1}=1$. Then an $A P$ factorization of $G$ can be rewritten as

$$
F_{+}\left[\begin{array}{cc}
1 & 0 \\
0 & G_{2}
\end{array}\right]=\Lambda G_{-}
$$


where $F_{+}=G_{+}^{-1} \in A P^{+}$. Denote $F_{+}=\left(f_{i j}\right)_{i, j=1}^{n}$. From (2.1),$e_{-\lambda_{j}} f_{j 1} \in A P^{-}$, so that

$$
\Omega\left(f_{j 1}\right) \subset\left[0, \lambda_{j}\right]
$$

In particular, $f_{j 1}=0$ for all $j$ (if there are any) such that $\lambda_{j}<0$. Rewriting (2.1) as

$$
\left[\begin{array}{cc}
1 & 0 \\
0 & G_{2}^{-1}
\end{array}\right] G_{+} \Lambda=G_{-}^{-1}
$$

we find similarly that

$$
\Omega\left(g_{1 j}\right) \subset\left[0,-\lambda_{j}\right]
$$

where $G_{+}=\left(g_{i j}\right)_{i, j=1}^{n}$. Therefore, $g_{1 j}=0$ for all $j$ (if there are any) such that $\lambda_{j}>0$. Observe also that $G_{+} F_{+}=I$ implies that $\sum_{j=1}^{n} g_{1 j} f_{j 1}=1$. Since for nonzero $\lambda_{j}$ at least one of the entries $g_{1 j}, f_{j 1}$ is equal to zero, the latter equality proves the existence of zero partial $A P$ indices $\lambda_{j}$. Due to (2.2), (2.3), the corresponding functions $g_{1 j}, f_{j 1}$ are constant, and for at least one value of $j, g_{1 j} f_{j 1} \neq 0$.

Applying an appropriate permutation of columns of $G_{+}$and rows of $G_{-}$, we may suppose without loss of generality that $\lambda_{1}=0, g_{11}=c \neq 0, f_{11}=d \neq 0$. Partitioning $G_{+}, F_{+}$as

$$
G_{+}=\left[\begin{array}{cc}
c & g_{1}^{+} \\
g_{2}^{+} & G_{2}^{+}
\end{array}\right], F_{+}=\left[\begin{array}{cc}
d & f_{1}^{+} \\
f_{2}^{+} & F_{2}^{+}
\end{array}\right],
$$

we conclude that $c=\operatorname{det} F_{2}^{+} / \operatorname{det} F_{+}=\operatorname{det} F_{2}^{+} \operatorname{det} G_{+}$. Since $c \neq 0$, the matrix $F_{2}^{+}$ is invertible in $A P^{+}$simultaneously with $G_{+}$. From (2.1) and (2.2) it follows that the left-upper entry of $G_{-}$and $H_{-}=G_{-}^{-1}$ equals $d$ and $c$, respectively. Thus,

$$
G_{-}=\left[\begin{array}{cc}
d & g_{1}^{-} \\
g_{2}^{-} & G_{2}^{-}
\end{array}\right], H_{-}=\left[\begin{array}{cc}
c & h_{1}^{-} \\
h_{2}^{-} & H_{2}^{-}
\end{array}\right]
$$

and $c=\operatorname{det} G_{2}^{-} / \operatorname{det} G_{-}$. Since $c \neq 0$, the matrix $G_{2}^{-}$is invertible in $A P^{-}$together with $G_{-}$. Now partition $\Lambda=\left[\begin{array}{cc}1 & 0 \\ 0 & \Lambda_{2}\end{array}\right]$. Then (2.1) yields $F_{2}^{+} G_{2}=\Lambda_{2} G_{2}^{-}$, or $G_{2}=\left(F_{2}^{+}\right)^{-1} \Lambda_{2} G_{2}^{-}$. Since $\left(F_{2}^{+}\right)^{ \pm 1} \in A P^{+}$and $\left(G_{2}^{-}\right)^{ \pm 1} \in A P^{-}$, the latter formula delivers an $A P$ factorization of $G_{2}$. This proves the desired statement in the case $G_{1}=1$.

If $G_{1}=e_{\lambda}$, then the matrix $e_{-\lambda} G=\operatorname{diag}\left[1, e_{-\lambda} G_{2}\right]$ is $A P$ factorable together with $G$. According to the already proven particular case, $e_{-\lambda} G_{2}$ is $A P$ factorable. But then $G_{2}$ is $A P$ factorable as well.

An induction argument allows us to consider $G_{1}$ of the form $\operatorname{diag}\left[e_{\lambda_{1}}, \ldots, e_{\lambda_{k}}\right]=$ $\Lambda_{1}$. Finally, for an arbitrary $A P$ factorable $G_{1}=G_{+}^{(1)} \Lambda_{1} G_{-}^{(1)}$ we can write

$$
G=\operatorname{diag}\left[G_{+}^{(1)}, I\right] \operatorname{diag}\left[\Lambda_{1}, G_{2}\right] \operatorname{diag}\left[G_{-}^{(1)}, I\right]
$$

and consider an (AP factorable) matrix $\operatorname{diag}\left[\Lambda_{1}, G_{2}\right]$ instead of the original matrix $G$.

Another technical tool we need applies to matrix functions $G_{f}$ with a trinomial $f$ containing an invertible $c_{-1}$ coefficient. 
Lemma 2.2. Let $G$ be of the form (1.4) with $f$ given by (1.5). If $c_{-1}$ is invertible, then $G$ is $A P\left(A P_{W}\right)$ factorable only simultaneously with (and has the same partial $A P$ indices as) the matrix function

$$
G_{1}=\left[\begin{array}{cc}
e_{\lambda_{1}} I_{m} & 0 \\
f_{1} & e_{-\lambda_{1}} I_{m}
\end{array}\right]
$$

where

$$
\begin{gathered}
f_{1}=c_{-1}^{(1)} e_{-\nu_{1}}+c_{0}^{(1)}+c_{1}^{(1)} e_{\alpha_{1}}, \\
c_{-1}^{(1)}=(-1)^{s}\left(c_{-1}^{-1} c_{0}\right)^{s+1}, c_{0}^{(1)}=c_{-1}^{-1} c_{1}, c_{1}^{(1)}=(-1)^{s+1}\left(c_{-1}^{-1} c_{0}\right)^{s+2}, \\
\lambda_{1}=\nu, \nu_{1}=\alpha-s \nu, \alpha_{1}=(s+1) \nu-\alpha,
\end{gathered}
$$

and finally, $s$ is the integral part of $\frac{\alpha}{\nu}: s \in \mathbb{Z}$ and $s<\frac{\alpha}{\nu}<s+1$.

Proof. It suffices to construct matrix functions $X_{+}$and $X_{-}$such that $X_{+}^{ \pm 1} \in A P_{W}^{+}$, $X_{-}^{ \pm 1} \in A P_{W}^{-}$and

$$
X_{+} G X_{-}=G_{1}
$$

To this end, let

$$
\begin{aligned}
& X_{+}=\left[\begin{array}{cc}
c_{-1}^{-1} f e_{\nu} & -e_{\lambda+\nu} I \\
e_{-\lambda-\nu} I+\left(g-e_{-\lambda} I\right) c_{-1}^{-1} f & I-g e_{\lambda}
\end{array}\right]\left[\begin{array}{cc}
I & 0 \\
0 & c_{-1}^{-1}
\end{array}\right], \\
& X_{-}=\left[\begin{array}{cc}
I & 0 \\
0 & c_{-1}
\end{array}\right]\left[\begin{array}{cc}
e_{-\alpha} I+\sum_{j=1}^{s}(-1)^{j}\left(c_{-1}^{-1} c_{0}\right)^{j} e_{j \nu-\alpha} & I \\
-I & 0
\end{array}\right],
\end{aligned}
$$

where $g=c_{-1}^{-1} c_{1}-\sum_{j=1}^{s+2}(-1)^{j}\left(c_{-1}^{-1} c_{0}\right)^{j} e_{(j-1) \nu-\alpha}$. Directly from the definition of $s$ it follows that $X_{-} \in A P^{-}$. Since $\operatorname{det} X_{-}=\operatorname{det} c_{-1}$ is a non-zero constant, $X_{-}^{-1}$ belongs to $A P^{-}$together with $X_{-}$.

A straightforward computation shows that

$$
\begin{aligned}
X_{+} G X_{-}= & {\left[\begin{array}{cc}
c_{-1}^{-1} f e_{\nu} & -e_{\lambda+\nu} I \\
e_{-\lambda-\nu} I+\left(g-e_{-\lambda} I\right) c_{-1}^{-1} f & I-g e_{\lambda}
\end{array}\right] } \\
& \times\left[\begin{array}{cc}
e_{\lambda} I & 0 \\
c_{-1}^{-1} f & e_{-\lambda} I
\end{array}\right]\left[\begin{array}{cc}
e_{-\alpha} I+\sum_{j=1}^{s}(-1)^{j}\left(c_{-1}^{-1} c_{0}\right)^{j} e_{j \nu-\alpha} & I \\
-I & 0
\end{array}\right] \\
= & {\left[\begin{array}{cc}
c_{-1}^{-1} f e_{\nu} & -e_{\lambda+\nu} I \\
e_{-\lambda-\nu} I+\left(g-e_{-\lambda} I\right) c_{-1}^{-1} f & I-g e_{\lambda}
\end{array}\right] } \\
& \times\left[\begin{array}{cc}
e_{\nu} I+\sum_{j=1}^{s}(-1)^{j}\left(c_{-1}^{-1} c_{0}\right)^{j} e_{(j+1) \nu} & e_{\lambda} I \\
c_{-1}^{-1} e_{-\alpha} f+c_{-1}^{-1} f \sum_{j=1}^{s}(-1)^{j}\left(c_{-1}^{-1} c_{0}\right)^{j} e_{j \nu-\alpha}-e_{-\lambda} I & c_{-1}^{-1} f
\end{array}\right] \\
= & \left(y_{i j}\right)_{i, j=1}^{2},
\end{aligned}
$$


where

$$
\begin{aligned}
y_{11}= & c_{-1}^{-1} f e_{2 \nu}+c_{-1}^{-1} f \sum_{j=1}^{s}(-1)^{j}\left(c_{-1}^{-1} c_{0}\right)^{j} e_{(j+2) \nu}-c_{-1}^{-1} f e_{2 \nu} \\
& -c_{-1}^{-1} f \sum_{j=1}^{s}(-1)^{j}\left(c_{-1}^{-1} c_{0}\right)^{j} e_{(j+2) \nu}+e_{\nu} I=e_{\nu} I, \\
y_{12}= & c_{-1}^{-1} f e_{\lambda+\nu}-c_{-1}^{-1} f e_{\lambda+\nu}=0, \\
y_{22}= & e_{-\nu} I+\left(g e_{\lambda}-I\right) c_{-1}^{-1} f+\left(I-g e_{\lambda}\right) c_{-1}^{-1} f=e_{-\nu} I,
\end{aligned}
$$

and finally,

$$
\begin{aligned}
y_{21}= & \left(e_{-\lambda-\nu} I+\left(g-e_{-\lambda} I\right) c_{-1}^{-1} f\right)\left(e_{\nu} I+\sum_{j=1}^{s}(-1)^{j}\left(c_{-1}^{-1} c_{0}\right)^{j} e_{(j+1) \nu}\right) \\
& +\left(I-g e_{\lambda}\right)\left(c_{-1}^{-1} e_{-\alpha} f+c_{-1}^{-1} f \sum_{j=1}^{s}(-1)^{j}\left(c_{-1}^{-1} c_{0}\right)^{j} e_{j \nu-\alpha}-e_{-\lambda} I\right) \\
= & e_{-\lambda} I+\sum_{j=1}^{s}(-1)^{j}\left(c_{-1}^{-1} c_{0}\right)^{j} e_{j \nu-\lambda}+\left(g-e_{-\lambda} I\right) \\
& \times\left(c_{-1}^{-1} f e_{\nu}+c_{-1}^{-1} f \sum_{j=1}^{s}(-1)^{j}\left(c_{-1}^{-1} c_{0}\right)^{j} e_{(j+1) \nu}\right. \\
& \left.\quad-c_{-1}^{-1} f e_{\nu}-c_{-1}^{-1} f \sum_{j=1}^{s}(-1)^{j}\left(c_{-1}^{-1} c_{0}\right)^{j} e_{(j+1) \nu}+I\right) \\
= & e_{-\lambda} I+\sum_{j=1}^{s}(-1)^{j}\left(c_{-1}^{-1} c_{0}\right)^{j} e_{j \nu-\lambda}+g-e_{-\lambda} I \\
= & \sum_{j=1}^{s}(-1)^{j}\left(c_{-1}^{-1} c_{0}\right)^{j} e_{j \nu-\lambda}+c_{-1}^{-1} c_{1}-\sum_{j=1}^{s+2}(-1)^{j}\left(c_{-1}^{-1} c_{0}\right)^{j} e_{(j-1) \nu-\alpha} \\
= & (-1)^{s}\left(c_{-1}^{-1} c_{0}\right)^{s+1} e_{s \nu-\alpha}+c_{-1}^{-1} c_{1}+(-1)^{s+1}\left(c_{-1}^{-1} c_{0}\right)^{s+2} e_{(s+1) \nu-\alpha}=f_{1} .
\end{aligned}
$$

This implies (2.6). Since $\operatorname{det} G=\operatorname{det} G_{1}=1$, from (2.6) it follows, in particular, that $\operatorname{det} X_{+}=\left(\operatorname{det} X_{-}\right)^{-1}$ is a non-zero constant. It remains to show that $X_{+} \in$ $A P^{+}$, because then $X_{+}^{-1} \in A P^{+}$as well. Three blocks of $X_{+}$are obviously in $A P^{+}$. The remaining (left-lower) block can be rewritten as

$$
\begin{aligned}
& e_{-\lambda-\nu} I+\left(g-e_{-\lambda} I\right) c_{-1}^{-1} f \\
&= e_{-\lambda-\nu} I+c_{-1}^{-1} c_{1} e_{-\nu}+c_{-1}^{-1} c_{1} c_{-1}^{-1} c_{0}+\left(c_{-1}^{-1} c_{1}\right)^{2} e_{\alpha} \\
& \quad-\sum_{j=1}^{s+2}(-1)^{j}\left(c_{-1}^{-1} c_{0}\right)^{j} e_{(j-2) \nu-\alpha}-\sum_{j=1}^{s+2}(-1)^{j}\left(c_{-1}^{-1} c_{0}\right)^{j+1} e_{(j-1) \nu-\alpha} \\
& \quad-\sum_{j=1}^{s+2}(-1)^{j}\left(c_{-1}^{-1} c_{0}\right)^{j} e_{(j-1) \nu}\left(c_{-1}^{-1} c_{1}\right)-e_{-\lambda-\nu} I-c_{-1}^{-1} c_{0} e_{-\lambda}-c_{-1}^{-1} c_{1} e_{-\nu} \\
&=c_{-1}^{-1} c_{1} c_{-1}^{-1} c_{0}+\left(c_{-1}^{-1} c_{1}\right)^{2} e_{\alpha}-\sum_{j=1}^{s+2}(-1)^{j}\left(c_{-1}^{-1} c_{0}\right)^{j} e_{(j-1) \nu}\left(c_{-1}^{-1} c_{1}\right) \\
& \quad+\left(c_{-1}^{-1} c_{0}\right) e_{-\lambda}-(-1)^{s}\left(c_{-1}^{-1} c_{0}\right)^{s+3} e_{(s+1) \nu-\alpha}-\left(c_{-1}^{-1} c_{0}\right) e_{-\lambda} .
\end{aligned}
$$


Cancelling out the terms $\pm\left(c_{-1}^{-1} c_{0}\right) e_{-\lambda}$ in the last expression, we see that this block belongs to $A P^{+}$as well.

Formula (2.6) is a particular case of the transformation introduced in 2] for an arbitrary $A P$ polynomial (not necessarily a trinomial) $f$ with invertible Fourier coefficient corresponding to the leftmost point in $\Omega(f) \cap(-\lambda, \lambda)$. However, in [2] only the case of commuting coefficients was considered. Also, formulas (2.7) for a trinomial case are more explicit than the general formulas of 2 .

The resulting matrix $G_{1}$ in general has the same structure as the original matrix $G: \Omega\left(f_{1}\right) \subset\left\{-\nu_{1}, 0, \alpha_{1}\right\}$, where $\alpha_{1}, \nu_{1}>0, \alpha_{1}+\nu_{1}=\lambda_{1}$ and $\beta_{1}=\nu_{1} / \alpha_{1}$ is irrational together with $\beta$. In some instances, however, $G_{1}$ may be easier to deal with. One such situation is discussed in the next theorem; other applications of Lemma 2.2 can be found in subsequent sections.

Theorem 2.3. Let the matrix $G$ be given by (1.4), (1.5) with $c_{-1}$ invertible, $c_{0} c_{-1}^{-1}$ nilpotent and having all Jordan cells of the size at most $\left[\frac{\alpha}{\nu}\right]+2$. Then 1) $G$ is $A P_{W}$ factorable, and 2) its partial AP indices equal zero if and only if $c_{1}$ is invertible.

Proof. Due to Lemma 2.2, we may consider the matrix (2.4) instead of $G$. The conditions imposed on the Jordan structure of $c_{0} c_{-1}^{-1}$ imply that $\left(c_{-1}^{-1} c_{0}\right)^{s+2}=0$. Thus, $f_{1}$ in (2.4) is in fact a binomial with $\Omega\left(f_{1}\right) \subset\left\{-\nu_{1}, 0\right\}$. According to Theorem 1.2 the matrix $G_{1}$ is $A P_{W}$ factorable, and its partial $A P$ indices equal zero if and only if the constant term $c_{-1}^{-1} c_{1}$ of $f_{1}$ is invertible. The latter condition is equivalent to the invertibility of $c_{1}$.

Recall now the duality between an $A P$ factorization (1.2) of $G_{f}$ and that of $G_{f^{*}}$ :

$$
G_{f^{*}}=\left(J G_{-}^{*}\right) \Lambda^{*}\left(G_{+}^{*} J\right),
$$

where $J=\left[\begin{array}{ll}0 & I \\ I & 0\end{array}\right]$. From (2.8) and Theorem 2.3 follows

Corollary 2.4. Let the matrix $G$ be given by (1.4), (1.5) with $c_{1}$ invertible, $c_{0} c_{1}^{-1}$ nilpotent and having all Jordan cells of the size at most $\left[\frac{\nu}{\alpha}\right]+2$. Then $G$ is $A P_{W}$ factorable, and its partial $A P$ indices equal zero if and only if $c_{-1}$ is invertible.

Observe that the condition on the size of Jordan cells is satisfied automatically if $m=2$. Hence, the following statement holds.

Corollary 2.5. Let the matrix $G$ be given by (1.4), (1.5) with $m=2$, let one of the coefficients $c_{ \pm 1}$ be non-singular, and let the corresponding product $c_{0} c_{ \pm 1}^{-1}$ be nilpotent. Then 1) $G$ is $A P_{W}$ factorable, and 2) its partial $A P$ indices equal zero if and only if the second of the coefficients $c_{ \pm 1}$ is invertible as well.

\section{MAIN RESUlT}

We now turn to matrices (1.4) with the off-diagonal block (1.5) having pairwise commuting coefficients $c_{ \pm 1}, c_{0}$. The representation (1.6) is not unique, and we choose one with the maximal possible number $r$ of diagonal blocks. Each triple $\left\{c_{-1, k}, c_{0 k}, c_{1 k}\right\}$ is then irreducible, that is, does not allow a further reduction to a block diagonal form with the help of a common similarity. Of course, the commutativity property of $\left\{c_{-1}, c_{0}, c_{1}\right\}$ is inherited by the triples $\left\{c_{-1, k}, c_{0 k}, c_{1 k}\right\}$.

The ambiguity of $T$ also allows us, for each $k=1, \ldots, r$, to put one of the matrices $c_{j k}$ (with our choice of $j=0, \pm 1$ ) in its Jordan canonical form. If, for a 
given $k$, at least one of the matrices $c_{j k}$ is unicellular (that is, its canonical Jordan form consists of only one cell), then for such a $T$ all the matrices $c_{j k}$ with the same $k$ automatically become upper triangular and, in addition, have a Toeplitz structure. The latter means that $(p, q)$-entry of each of the matrices $c_{-1, k}, c_{0, k}, c_{1, k}$ is the same as its $(p+1, q+1)$-entry $\left(p, q=1, \ldots, l_{k}-1\right)$. For $l_{k}>1$, the common value of the entries right above the main diagonal in $c_{j k}$ for such $k$ will be denoted by $\eta_{j k}$ (of course, the common value of the diagonal elements of the $c_{j k}$ in this case is $\left.\xi_{j k}\right)$.

With this notation at hand, we are ready to formulate our main result.

Theorem 3.1. Let $G$ be given by (1.4), (1.5) with pairwise commuting coefficients $c_{ \pm 1}, c_{0}$. Suppose that in (1.6) for each $k=1, \ldots, r$ at least one of the following conditions holds: 1) $\xi_{0 k} \neq 0$, 2) $\left.\xi_{1, k} \xi_{-1, k} \neq 0,3\right)$ one of the blocks $c_{ \pm 1, k}, c_{0 k}$ is unicellular, 4) $\left.l_{k} \leq 3,5\right) \xi_{1, k}$ or $\xi_{-1, k}$ differs from zero and $l_{k} \leq 4$. Then $G$ is not $A P$ factorable if, for at least one value of $k$,

$$
\left|\xi_{1, k}^{\nu} \xi_{-1, k}^{\alpha}\right|=\left|\xi_{0 k}\right|^{\lambda} \neq 0 \text {, or } \xi_{-1, k}=\xi_{0 k}=\xi_{1, k}=0 \text { and }\left|\eta_{1, k}^{\nu} \eta_{-1, k}^{\alpha}\right|=\left|\eta_{0 k}\right|^{\lambda} \neq 0,
$$

and is $A P_{W}$ factorable otherwise.

Proof. Using (1.6), introduce a matrix

$$
\left[\begin{array}{cc}
T^{-1} & 0 \\
0 & T^{-1}
\end{array}\right] G\left[\begin{array}{cc}
T & 0 \\
0 & T
\end{array}\right]=\left[\begin{array}{cc}
e_{\lambda} I_{m} & 0 \\
\operatorname{diag}\left[c_{-1, k} e_{-\nu}+c_{0 k}+c_{1, k} e_{\alpha}\right] & e_{-\lambda} I_{m}
\end{array}\right]
$$

having the same factorization properties as $G$. By an appropriate permutation of its rows and columns, this matrix can be further rewritten as a direct sum of the blocks

$$
G_{k}=\left[\begin{array}{cc}
e_{\lambda} I_{l_{k}} & 0 \\
c_{-1, k} e_{-\nu}+c_{0 k}+c_{1, k} e_{\alpha} & e_{-\lambda} I_{l_{k}}
\end{array}\right]
$$

$k=1, \ldots r$. Let $R=\{1, \ldots, r\}$ and denote by $R_{0}$ the subset of those $r \in R$ such that $\xi_{1, k}=\xi_{-1, k}=\xi_{0 k}=0, l_{k}>1$ and (at least) one of the blocks $c_{ \pm 1, k}, c_{0 k}$ is unicellular. We now partition $R$ into a disjoint union $\bigcup_{j=1}^{4} R_{j}$, where

$$
\begin{aligned}
& R_{1}=\left\{k:\left|\xi_{1, k}^{\nu} \xi_{-1, k}^{\alpha}\right|=\left|\xi_{0 k}\right|^{\lambda} \neq 0\right\}, \\
& R_{2}=\left\{k \in R_{0}:\left|\eta_{1, k}^{\nu} \eta_{-1, k}^{\alpha}\right|=\left|\eta_{0 k}\right|^{\lambda} \neq 0\right\}, \\
& R_{3}=R_{0} \backslash R_{2}, \\
& R_{4}=R \backslash\left(R_{1} \cup R_{0}\right) .
\end{aligned}
$$

For every $k \in R_{0}$, yet another permutation of rows and columns allows us to represent $G_{k}$ as a direct sum of $\left[\begin{array}{cc}e_{\lambda} & 0 \\ 0 & e_{-\lambda}\end{array}\right]$ with

$$
G_{k}^{\prime}=\left[\begin{array}{cc}
e_{\lambda} I_{l_{k}-1} & 0 \\
c_{-1, k}^{\prime} e_{-\nu}+c_{0 k}^{\prime}+c_{1, k}^{\prime} e_{\alpha} & e_{-\lambda} I_{l_{k}-1}
\end{array}\right] \text {. }
$$

Here $c_{j k}^{\prime}$ are obtained from $c_{j k}$ by deleting its first column and last row. The Toeplitz structure of $c_{j k}$ is inherited by $c_{j k}^{\prime}$. In particular, the $c_{j k}^{\prime}$ pairwise commute and $\sigma\left(c_{j k}^{\prime}\right)=\left\{\eta_{j k}\right\}\left(j=0, \pm 1 ; k \in R_{0}\right)$.

Denote by $G^{(1)}$ the direct sum of all the blocks $G_{k}, k \in R_{1}$, and $G_{k}^{\prime}, k \in R_{2}$. Let $G^{(2)}$ be a direct sum of all $G_{k}\left(k \in R_{4}\right), G_{k}^{\prime}\left(k \in R_{3}\right)$, and $\left|R_{2}\right|$ copies of 
the diagonal blocks $\left[\begin{array}{cc}e_{\lambda} & 0 \\ 0 & e_{-\lambda}\end{array}\right]$. Then $G$ can be put in the form $G^{(1)} \oplus G^{(2)}$ by an appropriate permutation of its rows and columns. In turn, $G^{(1)}$ will become a permutation of a matrix of the type (1.4) with $f=b_{-1} e_{-\nu}+b_{0}+b_{1} e_{\alpha}$ and $b_{j}=\left(\bigoplus_{k \in R_{1}} c_{j k}\right) \oplus\left(\bigoplus_{k \in R_{2}} c_{j k}^{\prime}\right)$.

In terms of the sets $R_{j}$, this theorem claims that $G$ is $A P_{W}$ factorable if $R_{1} \cup R_{2}=$ $\emptyset$, and is not $A P$ factorable otherwise. This follows from Lemma 2.1 provided that $G^{(2)}$ is $A P_{W}$ factorable and, for $R_{1} \cup R_{2} \neq \emptyset, G^{(1)}$ is not $A P$ factorable. The latter statement holds due to Corollary 1.4. It remains to prove the former. We will do this by showing that each direct summand of $G^{(2)}$ is $A P_{W}$ factorable. There are five types of these summands:

(i) diagonal blocks $\left[\begin{array}{cc}e_{\lambda} & 0 \\ 0 & e_{-\lambda}\end{array}\right]$,

and matrices (1.4) with $f$ given by (1.5), pairwise commuting $c_{ \pm 1}, c_{0}$ (slightly abusing the notation, we again denote their size by $m)$, singleton spectra $\sigma\left(c_{j}\right)=$ $\left\{\xi_{j}\right\}(j= \pm 1,0)$ for which

(ii) $\left|\xi_{1}^{\nu} \xi_{-1}^{\alpha}\right| \neq\left|\xi_{0}\right|^{\lambda}$

(iii) $\xi_{0}=0$, exactly one of $\xi_{ \pm 1}$ differs from zero and (at least) one of the blocks $c_{ \pm 1}, c_{0}$ is unicellular,

(iv) $\xi_{0}=0$, exactly one of $\xi_{ \pm 1}$ differs from zero, and $m \leq 4$,

(v) $\xi_{0}=\xi_{1}=\xi_{-1}=0$ and $m \leq 3$.

Indeed, the blocks $G_{k}$ with $k \in R_{1}$ have no impact on $G^{(2)}, k \in R_{2}$ generate only summands of type (i), $k \in R_{3}$ yield summands of type (i) and (ii) or (iii), and $k \in R_{4}$ produce summands of types (ii)-(v).

The summands of type (i) are trivially $A P_{W}$ factorable (with partial $A P$ indices $\pm \lambda$ ). The summands of type (ii) are $A P_{W}$ factorable (with zero partial $A P$ indices) according to Theorem 1.3. It remains to consider matrices (1.4) of types (iii)-(v).

In cases (iii) and (iv) we may without loss of generality suppose that $\xi_{1}=0$, $\xi_{-1} \neq 0$; otherwise, $G_{f *}$ can be considered instead of $G_{f}$. If in addition, $c_{0}=0$ or $c_{1}=0$, then $f$ is a binomial and the corresponding matrix (1.4) is $A P_{W}$ factorable due to Theorem 1.2 This happens, in particular, if $m=1$.

If all three coefficients of $f$ differ from zero, we consider the matrix 2.4). It can happen that $c_{0}^{s+2}=0$, in which case the resulting block (2.5) is a binomial. Applying Theorem 1.2 and Lemma 2.2 we conclude that (2.4), and therefore (1.4), are $A P_{W}$ factorable. If $c_{0}^{s+2} \neq 0$, we consider cases (iii) and (iv) separately.

(iii) The matrices $c_{j}$ have an upper triangular Toeplitz structure which is inherited by the coefficients $c_{j}^{(1)}$ of (2.5). Hence,

$$
m>\operatorname{rank} c_{0}^{(1)}=\operatorname{rank} c_{1}
$$

and

$$
m>\operatorname{rank} c_{-1}^{(1)}=\operatorname{rank} c_{0}^{s+1}>\operatorname{rank} c_{1}^{(1)}=\operatorname{rank} c_{0}^{s+2}>0 .
$$

Let $q=\max \left\{\operatorname{rank} c_{0}^{(1)}, \operatorname{rank} c_{-1}^{(1)}\right\}, p=m-q$. Then both $p$ and $q$ are strictly positive. By a permutation of its rows and columns, the matrix $G_{1}$ can be reduced to the form

$$
\left[\begin{array}{cc}
e_{\nu} I_{p} & 0 \\
0 & e_{-\nu} I_{p}
\end{array}\right] \oplus\left[\begin{array}{cc}
e_{\nu} I_{q} & 0 \\
f_{2} & e_{-\nu} I_{q}
\end{array}\right]
$$


where

$$
f_{2}=c_{-1}^{(2)} e_{-\nu_{1}}+c_{0}^{(2)}+c_{1}^{(2)} e_{\alpha_{1}}
$$

and the matrices $c_{j}^{(2)}$ are obtained from $c_{j}^{(1)}$ by deleting their first $p$ columns and last $p$ rows. It suffices to prove now that the second direct summand in 3.2 is $A P_{W}$ factorable.

If rank $c_{0}^{(1)} \geq \operatorname{rank} c_{-1}^{(1)}$, this summand falls into type (ii). In the opposite case, this is again a matrix of type (iii), but its size is strictly smaller than that of the original matrix: $q<m$. By induction we now conclude that all matrices of type (iii) are $A P_{W}$ factorable.

(iv) The case of unicellular $c_{0}$ is covered by (iii). Since $m \leq 4$ and $c_{0}^{s+2} \neq 0$, the only remaining case is $s=0, m=4$ and $c_{0}$ consisting of one $3 \times 3$ and one $1 \times 1$ Jordan cell. The same Jordan structure is possessed by the matrix $c_{-1}^{-1} c_{0}$. Without loss of generality we may suppose that in (2.5)

$$
c_{-1}^{(1)}=c_{-1}^{-1} c_{0}=\left[\begin{array}{cccc}
0 & 1 & 0 & 0 \\
0 & 0 & 1 & 0 \\
0 & 0 & 0 & 0 \\
0 & 0 & 0 & 0
\end{array}\right] \text {. }
$$

Then

$$
c_{1}^{(1)}=-\left(c_{-1}^{-1} c_{0}\right)^{2}=\left[\begin{array}{cccc}
0 & 0 & -1 & 0 \\
0 & 0 & 0 & 0 \\
0 & 0 & 0 & 0 \\
0 & 0 & 0 & 0
\end{array}\right] \text {. }
$$

The matrix $c_{0}^{(1)}=c_{-1}^{-1} c_{1}$ is nilpotent and commutes with (3.4). Thus,

$$
c_{0}^{(1)}=\left[\begin{array}{llll}
0 & z & u & b \\
0 & 0 & z & 0 \\
0 & 0 & 0 & 0 \\
0 & 0 & a & 0
\end{array}\right]
$$

If $a=b=0$, then the matrix $G_{1}$ can be split into a direct sum of $\left[\begin{array}{cc}e_{\nu} I_{2} & 0 \\ 0 & e_{-\nu} I_{2}\end{array}\right]$ and $G_{2}=\left[\begin{array}{cc}e_{\nu} I_{2} & 0 \\ f_{2} & e_{-\nu} I_{2}\end{array}\right]$, where $f_{2}$ is given by (3.3) with

$$
c_{-1}^{(2)}=I_{2}, c_{0}^{(2)}=\left[\begin{array}{cc}
z & u \\
0 & z
\end{array}\right], c_{1}^{(2)}=\left[\begin{array}{cc}
0 & -1 \\
0 & 0
\end{array}\right] .
$$

The matrix $G_{2}$ is of type (ii) or (iii) (depending on whether or not $z$ is zero), and therefore $A P_{W}$ factorable. Of course, $G_{1}$ is $A P_{W}$ factorable together with $G_{2}$.

If $a$ or $b$ differs from zero, represent $G_{1}$ as a direct sum of $\operatorname{diag}\left[e_{\nu}, e_{-\nu}\right]$ with $G_{3}=\left[\begin{array}{cc}e_{\nu} I_{3} & 0 \\ f_{3} & e_{-\nu} I_{3}\end{array}\right]$, where $f_{3}=c_{-1}^{(3)} e_{-\nu_{1}}+c_{0}^{(3)}+c_{1}^{(3)} e_{\alpha_{1}}$ and

$$
c_{-1}^{(3)}=\left[\begin{array}{lll}
1 & 0 & 0 \\
0 & 1 & 0 \\
0 & 0 & 0
\end{array}\right], c_{0}^{(3)}=\left[\begin{array}{ccc}
z & u & b \\
0 & z & 0 \\
0 & a & 0
\end{array}\right], c_{1}^{(3)}=\left[\begin{array}{ccc}
0 & -1 & 0 \\
0 & 0 & 0 \\
0 & 0 & 0
\end{array}\right] .
$$

The explicit $A P_{W}$ factorization of $G_{3}$ is shown in Appendix A of the supplement. Hence, all matrices of type (iv) are $A P_{W}$ factorable. 
Finally, consider the remaining type (v). If $m \leq 2$, then each matrix $c_{j}$ either is unicellular or equals zero. In both cases, an $A P_{W}$ factorization exists. Therefore, we may suppose that $m=3$. Excluding another trivial case $c_{0}=0$ (in which $f$ is a binomial), we are left with the only possible Jordan structure of $c_{0}$ : one $2 \times 2$ and one $1 \times 1$ block. Then, without loss of generality,

$$
c_{0}=\left[\begin{array}{lll}
0 & 1 & 0 \\
0 & 0 & 0 \\
0 & 0 & 0
\end{array}\right]
$$

The matrices $c_{ \pm 1}$ commute with $c_{0}$ and are nilpotent. Therefore,

$$
c_{ \pm 1}=\left[\begin{array}{ccc}
0 & y_{ \pm} & x_{ \pm} \\
0 & 0 & 0 \\
0 & z_{ \pm} & 0
\end{array}\right]
$$

The matrix $G$ splits into a direct sum of $\operatorname{diag}\left[e_{\lambda}, e_{-\lambda}\right]$ and $G_{1}=\left[\begin{array}{cc}e_{\lambda} I_{2} & 0 \\ f_{1} & e_{-\lambda} I_{2}\end{array}\right]$, where $f_{1}=c_{-1}^{(1)} e_{-\nu}+c_{0}^{(1)}+c_{1}^{(1)} e_{\alpha}$

$$
c_{0}^{(1)}=\left[\begin{array}{ll}
0 & 1 \\
0 & 0
\end{array}\right], \quad c_{ \pm 1}^{(1)}=\left[\begin{array}{cc}
x_{ \pm} & y_{ \pm} \\
0 & z_{ \pm}
\end{array}\right] .
$$

From commutativity of $c_{1}$ with $c_{-1}$ it follows that $x_{+} z_{-}=x_{-} z_{+}$; however, later on we will encounter a factorization problem for matrices $G_{1}$ with $c_{ \pm 1}^{(1)}$ not satisfying this requirement. Therefore, we do not impose the condition $x_{+} z_{-}=x_{-} z_{+}$in our consideration.

The case $x_{+}=x_{-}=z_{+}=z_{-}=0$ is excluded because otherwise the triple $\left\{c_{-1}, c_{0}, c_{1}\right\}$ would be reducible. The cases $x_{+} z_{+} \neq 0$ and $x_{-} z_{-} \neq 0$ are covered by Corollary 2.5. In all the remaining cases an $A P_{W}$ factorization of $G_{1}$ also exists; it is constructed explicitly in Appendix B of the supplement. Hence, matrices $G$ of type (v) are also $A P_{W}$ factorable.

As an application of Theorem 3.1 consider a difference equation

$$
c_{-1} y(t-\nu)+c_{0} y(t)+c_{1} y(t+\alpha)=g(t) \text { a.e. on }(0, \lambda),
$$

where $g$ is a given vector function in $L^{p}(0, \lambda), y$ is an unknown vector function in $L^{p}(\mathbb{R})$ with supp $y \subset[0, \lambda]$.

According to standard terminology, we say that (3.5) is normally solvable (in $L^{p}$ ) if the set of vector functions $g$ for which (3.5) has a solution is closed.

Theorem 3.2. In (3.5) let $\alpha+\nu=\lambda$, let $\frac{\alpha}{\nu}(>0)$ be irrational, and let the coefficients $c_{j} \in \mathbb{C}^{m \times m}$ satisfy the conditions of Theorem 3.1. Then the equation 3.5 is normally solvable if and only if, in the notation of Theorem 3.1, condition (3.1) fails for every $k$.

This result does not depend on $p \in(1, \infty)$.

Proof. As follows from [7, Section 4.1], equation (3.5) is normally solvable if and only if the Wiener-Hopf operator $W_{G}$, the symbol $G$ of which is given by (1.4), (1.5), has closed range in $L^{p}(0, \infty)$.

If condition (3.1) fails for all $k$, then the matrix function $G$ is $A P_{W}$ factorable due to Theorem [3.1] Hence, $W_{G}$ has a generalized inverse, and therefore its range is closed. 
To prove the converse statement, consider first a particular case when in (1.5) each matrix $c_{j}$ has a singleton spectrum $\left\{\xi_{j}\right\}$, and

$$
\left|\xi_{1}^{\nu} \xi_{-1}^{\alpha}\right|=\left|\xi_{0}\right|^{\lambda} \neq 0
$$

According to Theorem 3.1 the matrix function $G$ in this case is not $A P$ factorable.

If $m=1$, the homogeneous equation (3.5) takes the form

$$
y(t)= \begin{cases}-\frac{\xi_{-1}}{\xi_{0}} y(t-\nu) & \text { if } \nu<t<\lambda, \\ -\frac{\xi_{1}}{\xi_{0}} y(t+\alpha) & \text { if } 0<t<\nu,\end{cases}
$$

and has at most one linearly independent solution (see, for example, [4]).

For $m>1$, a similarity can be used to put the $c_{j}$ simultaneously in a triangular form, with $\xi_{j}$ on the diagonal. Therefore, the number of linearly independent solutions of the respective homogeneous equation (3.5) is at most $m$. Suppose that this equation is normally solvable. Then the corresponding Wiener-Hopf operator $W_{G}$ has a closed range and a finite dimensional kernel; in other words, it is $n$-normal. This property, as well as the index ind $W_{G}$ of the operator $W_{G}$ (the difference between the dimension of its kernel and the codimension of its range), is preserved under small perturbations. Consider such a small perturbation $W_{G_{f^{\prime}}}$ with $f^{\prime}=$ $c_{-1} e_{-\nu}+\left(c_{0}+\epsilon I\right)+c_{1} e_{\alpha}$, and $0 \neq\left|\xi_{0}+\epsilon\right| \neq\left|\xi_{0}\right|$. Then $G^{\prime}=G_{f^{\prime}}$ admits an $A P_{W}$ factorization with zero partial $A P$ indices (Corollary 1.4), so that $W_{G^{\prime}}$ is invertible. Hence, ind $W_{G}=$ ind $W_{G^{\prime}}=0$. From here it follows that codim $\operatorname{Im} W_{G}$ is finite together with $\operatorname{dim} \operatorname{Ker} W_{G}$; that is, the operator $W_{G}$ is Fredholm. Since $G \in A P_{W}$, Theorem 2.5 of [7] implies that $G$ is $A P_{W}$ factorable. This contradiction shows that in fact the range $\operatorname{Im} W_{G}$ of the operator $W_{G}$ is not closed.

Finally, consider the general case when (3.1) holds for some $k$. Then, as was shown in the proof of Theorem 3.1, the corresponding matrix $G$ can be split into a direct sum of summands, a non-zero number of which are of the type just considered. Hence, $W_{G}$ also splits into a direct sum of operators, some of which have a nonclosed range. Therefore, $\operatorname{Im} W_{G}$ is not closed.

Remark. The above reasoning shows that for matrix functions $G$ satisfying the conditions of Theorem 3.1 the operator $W_{G}$ has a closed range if and only if $G$ is $A P$ factorable. This is not true in general; examples of not $A P$ factorable $2 \times 2$ triangular matrix functions $G \in A P_{W}$ for which $\operatorname{Im} W_{G}$ is closed can be found in [10].

\section{REMARKS ON $4 \times 4$ CASES}

Theorem 3.1 covers all matrices (1.4), (1.5) with commuting $c_{j}$ of size $m \leq 3$. Hence, the case of reducible $4 \times 4$ triples is also covered. For irreducible $\left\{c_{-1}, c_{0}, c_{1}\right\}$, each $c_{j}$ has a singleton spectrum, say $\sigma\left(c_{j}\right)=\left\{\xi_{j}\right\}$. The cases when at least one of the $\xi_{j}$ differs from zero or $c_{j}$ is unicellular also fall into the setting of Theorem 3.1

This leaves us with the situation of an irreducible triple of $4 \times 4$ nilpotent matrices $c_{j}(j=0, \pm 1)$, none of which is unicellular. We may suppose in addition that none of them is diagonalizable (that is, has only $1 \times 1$ Jordan cells). Indeed, a diagonalizable nilpotent matrix equals zero, and the corresponding $G$ is then $A P_{W}$ factorable due to Theorem 1.2. There remain three possible Jordan structures: two $2 \times 2$ cells, one $2 \times 2$ and two $1 \times 1$ cells, and one $3 \times 3$ and one $1 \times 1$ cells.

The following example demonstrates why the case of two $2 \times 2$ Jordan cells is hard to handle. 
Example. Let $c_{j}=\left[\begin{array}{cc}0 & c_{j}^{(0)} \\ 0 & 0\end{array}\right]$, where the $c_{j}^{(0)}$ are arbitrary (not necessarily commuting) non-singular $2 \times 2$ matrices, $j= \pm 1,0$. Then $G$ can be split into a direct sum of $\left[\begin{array}{cc}e_{\lambda} I_{2} & 0 \\ 0 & e_{-\lambda} I_{2}\end{array}\right]$ and $G_{0}=\left[\begin{array}{cc}e_{\lambda} I_{2} & 0 \\ c_{-1}^{(0)} e_{-\nu}+c_{0}^{(0)}+c_{1}^{(0)} e_{\alpha} & e_{-\lambda} I_{2}\end{array}\right]$. According to Lemma 2.1 the matrices $G$ and $G_{0}$ are $A P$ factorable only simultaneously. Hence, the $A P$ factorization problem for $G$ is reduced to the corresponding problem for matrices of the form (1.4) with non-commuting coefficients of $f$. Since the latter problem is still open, it is not surprising that a complete description of the $A P$ factorability for matrices (1.4), (1.5) with commuting $4 \times 4$ coefficients $c_{j}$ is also missing.

We will now discuss the two remaining possibilities for the Jordan structure of $c_{0}$. First, let $c_{0}$ consist of one $2 \times 2$ and two $1 \times 1$ Jordan cells. Without loss of generality, $c_{0}$ itself is in a Jordan form:

$$
c_{0}=\left[\begin{array}{llll}
0 & 1 & 0 & 0 \\
0 & 0 & 0 & 0 \\
0 & 0 & 0 & 0 \\
0 & 0 & 0 & 0
\end{array}\right] .
$$

From the commutativity of $c_{ \pm 1}$ with $c_{0}$ and their nilpotency it follows that

$$
c_{ \pm 1}=\left[\begin{array}{cccc}
0 & a_{ \pm} & b_{ \pm} & d_{ \pm} \\
0 & 0 & 0 & 0 \\
0 & f_{ \pm} & h_{ \pm} & l_{ \pm} \\
0 & g_{ \pm} & j_{ \pm} & k_{ \pm}
\end{array}\right],
$$

where $A_{ \pm}=\left[\begin{array}{cc}h_{ \pm} & l_{ \pm} \\ j_{ \pm} & k_{ \pm}\end{array}\right]$are themselves nilpotent.

We may also use a similarity to reduce $A_{+}$to its Jordan canonical form without disturbing $c_{0}$ and the structure of $A_{-}$. Thus, $h_{+}=k_{+}=j_{+}=0$ and $l_{+}=0$ or 1 .

If $l_{+}=1$, then commutativity of $c_{1}$ with $c_{-1}$ implies that $h_{-}=k_{-}=j_{-}=0$. If $l_{+}=0$ (that is, $A_{+}=0$ ), then we can use a similarity to reduce $A_{-}$to its Jordan canonical form without changing $c_{0}$ and $A_{+}$. Hence, in any case it may be supposed that $h_{ \pm}=k_{ \pm}=j_{ \pm}=0$, that is,

$$
c_{ \pm 1}=\left[\begin{array}{cccc}
0 & a_{ \pm} & b_{ \pm} & d_{ \pm} \\
0 & 0 & 0 & 0 \\
0 & f_{ \pm} & 0 & l_{ \pm} \\
0 & g_{ \pm} & 0 & 0
\end{array}\right] .
$$

Also, from commutativity of $c_{1}$ with $c_{-1}$ (which is preserved under the similarities applied above),

$$
l_{+} g_{-}=l_{-} g_{+}, l_{+} b_{-}=l_{-} b_{+}, b_{+} f_{-}+d_{+} g_{-}=b_{-} f_{+}+d_{-} g_{+} .
$$

Theorem 4.1. Let $G$ be given by (1.4), (1.5) with $c_{0}, c_{ \pm 1}$ as in (4.1) and (4.2), respectively, satisfying (4.3) and forming an irreducible triple $\left\{c_{-1}, c_{0}, c_{1}\right\}$. Then $G$ is not AP factorable if

$$
b_{+}=b_{-}=g_{+}=g_{-}=0, \quad\left|D_{-}^{\alpha} D_{+}^{\nu}\right|=\left|l_{+}^{\nu} l_{-}^{\alpha}\right| \neq 0,
$$


where

$$
D_{ \pm}=\operatorname{det}\left[\begin{array}{cc}
a_{ \pm} & d_{ \pm} \\
f_{ \pm} & l_{ \pm}
\end{array}\right]=a_{ \pm} l_{ \pm}-d_{ \pm} f_{ \pm}
$$

and is $A P_{W}$ factorable otherwise.

Proof. We need to show that $G$ is $A P_{W}$ factorable if

$$
\begin{aligned}
& \text { i) at least one of the numbers } b_{ \pm}, d_{ \pm} \text {differs from zero, or } \\
& \text { ii) } b_{+}=b_{-}=g_{+}=g_{-}=l_{+} l_{-} D_{+} D_{-}=0
\end{aligned}
$$

and that in the case

$$
\text { iii) } b_{+}=b_{-}=g_{+}=g_{-}=0, l_{ \pm} D_{ \pm} \neq 0
$$

it is $A P\left(A P_{W}\right)$ factorable if and only if

$$
\left|D_{-}^{\alpha} D_{+}^{\nu}\right| \neq\left|l_{+}^{\nu} l_{-}^{\alpha}\right| .
$$

In case i), rewrite $G$ as a direct sum of $\operatorname{diag}\left[e_{\lambda}, e_{-\lambda}\right]$ and another matrix of the form (1.4), with $m=3$ and

$$
c_{ \pm 1}=\left[\begin{array}{ccc}
a_{ \pm} & b_{ \pm} & d_{ \pm} \\
f_{ \pm} & 0 & l_{ \pm} \\
g_{ \pm} & 0 & 0
\end{array}\right], \quad c_{0}=\left[\begin{array}{ccc}
1 & 0 & 0 \\
0 & 0 & 0 \\
0 & 0 & 0
\end{array}\right] .
$$

If $c_{-1}$ is invertible, that is, $b_{-} g_{-} l_{-} \neq 0$, then Lemma 2.2 can be used. A direct computation shows that

$$
c_{-1}^{-1} c_{0}=\left[\begin{array}{ccc}
0 & 0 & 0 \\
\frac{1}{b_{-}} & 0 & 0 \\
0 & 0 & 0
\end{array}\right],
$$

and therefore $\left(c_{-1}^{-1} c_{0}\right)^{2}=0$. Hence, $f_{1}$ in (2.4) is at most a binomial, and the matrix $G_{1}$ is $A P_{W}$ factorable due to Theorem 1.2 The original matrix $G$ is then also $A P_{W}$ factorable.

Using (2.8) and appropriate transpositions of rows and columns, we can cover the case of invertible $c_{1}$, that is, $b_{+} g_{+} l_{+} \neq 0$. It remains to construct an $A P_{W}$ factorization in the cases when, in addition to (4.3),

$$
b_{+} g_{+} l_{+}=b_{-} g_{-} l_{-}=0 \text {. }
$$

This is done in Appendix C.

In cases ii) and iii), we represent $G$ as a direct sum of $\left[\begin{array}{cc}e_{\lambda} I_{2} & 0 \\ 0 & e_{-\lambda} I_{2}\end{array}\right]$ and another matrix $G_{1}$ of the form (1.4), (1.5) with $m=2$ and

$$
c_{ \pm 1}^{(1)}=\left[\begin{array}{cc}
a_{ \pm} & d_{ \pm} \\
f_{ \pm} & l_{ \pm}
\end{array}\right], \quad c_{0}^{(1)}=\left[\begin{array}{ll}
1 & 0 \\
0 & 0
\end{array}\right] .
$$

If $l_{+}=0$ and $d_{+} f_{+} \neq 0$, then the matrix $G_{1}$ is $A P_{W}$ factorable due to Corollary 2.5. The same reasoning applies if $l_{-}=0, d_{-} f_{-} \neq 0$. The cases $l_{+}=l_{-}=$ $d_{+} f_{+}=d_{-} f_{-}=0$ when not all of the four entries $d_{ \pm}, f_{ \pm}$equal zero are covered by Appendix B in the supplement. Observe that the case $d_{ \pm}=f_{ \pm}=0$ is excluded due to the irreducibility of the original triple $\left\{c_{-1}, c_{0}, c_{1}\right\}$ given by (4.1), (4.2). Hence, the situation when $l_{+}=l_{-}=0$ is covered completely.

In all other cases (when at least one of $l_{+}, l_{-}$differs from zero) we may use the symmetry (2.8) to suppose without loss of generality that, say, $l_{-} \neq 0$. An obvious 
similarity performed on the original $4 \times 4$ matrices $c_{ \pm 1}$ (and not changing $c_{0}$ ) allows us to suppose in addition that $d_{-}=f_{-}=0$. This similarity may, of course, change the values of $a_{ \pm}$and $d_{+}, f_{+}$; however, $\operatorname{det} c_{ \pm 1}^{(1)}$ remain the same, so that the new value of $a_{-}$is $D_{-} / l_{-}$. To simplify the notation, we redenote $D_{+}$by $D$.

If $l_{+}=0$, then $d_{+}, f_{+}$do not change under the above mentioned similarity. The only situation left uncovered by previous considerations is the case in which exactly one of $d_{+}, f_{+}$differs from zero.

In case ii), we are left with only two possibilities: 1) $l_{-} \neq 0, l_{+}=d_{-}=f_{-}=0$, exactly one of the entries $d_{+}, f_{+}$differs from zero, and 2) $l_{+} l_{-} \neq 0, d_{-}=f_{-}=0$, $a_{-} D=0$. Appendix $\mathrm{D}$ in the supplement shows that the corresponding matrix $G_{1}$ (and therefore $G$ ) is $A P_{W}$ factorable.

In case iii), the additional condition $d_{-}=f_{-}=0$ means that $a_{-}\left(=D_{-} / l_{-}\right) \neq 0$, and (4.4) can be rewritten as

$$
\left|a_{-}^{\alpha} D^{\nu}\right| \neq\left|l_{+}^{\nu}\right|
$$

A straightforward calculation shows that $G_{1}=X_{+} G^{\prime} X_{-}$, where

$$
X_{+}=\left[\begin{array}{cccc}
1 & d_{+} l_{-} e_{\lambda} & 0 & 0 \\
-\frac{f_{+}}{l_{+}} & a_{-} l_{+} e_{\lambda}-l_{-}\left(e_{\nu}+a_{-}\right) & -e_{\nu} & \frac{f_{+} e_{\nu}}{l_{+}} \\
0 & d_{+}\left(a_{-} l_{+}+a_{+} l_{-}\right) e_{\alpha} & -d_{+} & a_{+} \\
0 & \left(a_{-} l_{+}^{2}+d_{+} f_{+} l_{-}\right) e_{\alpha}-l_{-} l_{+} & -l_{+} & f_{+}
\end{array}\right]
$$

is invertible in $A P_{W}^{+}$,

$$
X_{-}=\left[\begin{array}{cccc}
1 & -\frac{d_{+} l_{-}}{a_{-} l_{+}} & 0 & -\frac{d_{+} e_{-\alpha}}{a_{-} l_{+}} \\
0 & \frac{1}{a_{-} l_{+}} & 0 & \frac{e_{-\alpha}}{a_{-} l_{+} l_{-}} \\
\frac{f_{+}\left(1+a_{-} e_{-\nu}\right)}{D} & -\frac{a_{+} l_{-}\left(1+a_{-} e_{-\nu}\right)}{a_{-} D} & \frac{f_{+} e_{-\lambda}}{D} & \frac{1}{l_{-}}-\frac{a_{+}\left(a_{-} e_{-\lambda}+e_{-\alpha}\right)}{a_{-} D} \\
0 & 0 & \frac{l_{+}}{D} & 0
\end{array}\right] .
$$

is invertible in $A P_{W}^{-}$, and

$$
G^{\prime}=\left[\begin{array}{cccc}
e_{\lambda} & 0 & 0 & 0 \\
0 & 1 & 0 & 0 \\
0 & 0 & 1 & 0 \\
a_{-} l_{+} e_{-\nu}+l_{+}+D e_{\alpha} & 0 & 0 & e_{-\lambda}
\end{array}\right]
$$

can be split into a direct sum of $I_{2}$ with

$$
G_{2}=\left[\begin{array}{cc}
e_{\lambda} & 0 \\
a_{-} l_{+} e_{-\nu}+l_{+}+D e_{\alpha} & e_{-\lambda}
\end{array}\right] .
$$

Of course, $G_{1}$ is $A P\left(A P_{W}\right)$ factorable only simultaneously with $G^{\prime}$, and in turn, $G^{\prime}$ has the same factorability properties as $G_{2}$. The latter matrix satisfies the conditions of Corollary 1.4 with $m=1$. In the notation of this statement, $\xi_{1, k}=D$, $\xi_{0 k}=l_{+}$and $\xi_{-1, k}=a_{-} l_{+}$with the only value of $k(=1)$, so that condition (1.7), necessary and sufficient for an $A P\left(A P_{W}\right)$ factorization to exist, is equivalent to (4.6).

Finally, let $c_{0}$ consist of one $3 \times 3$ and one $1 \times 1$ Jordan cells

$$
c_{0}=\left[\begin{array}{llll}
0 & 1 & 0 & 0 \\
0 & 0 & 1 & 0 \\
0 & 0 & 0 & 0 \\
0 & 0 & 0 & 0
\end{array}\right]
$$


Then the only possible form of $c_{ \pm 1}$ is

$$
c_{ \pm 1}=\left[\begin{array}{cccc}
0 & d_{ \pm} & f_{ \pm} & b_{ \pm} \\
0 & 0 & d_{ \pm} & 0 \\
0 & 0 & 0 & 0 \\
0 & 0 & a_{ \pm} & 0
\end{array}\right]
$$

where

$$
a_{+} b_{-}=a_{-} b_{+} .
$$

The case $a_{+}=a_{-}=b_{+}=b_{-}=0$ is excluded if the triple $\left\{c_{-1}, c_{0}, c_{1}\right\}$ is irreducible. Splitting $G$ into a direct sum of $\operatorname{diag}\left[e_{\lambda}, e_{-\lambda}\right]$ and another matrix of the form (1.4), we may suppose that $m=3$ and

$$
c_{ \pm 1}=\left[\begin{array}{ccc}
d_{ \pm} & f_{ \pm} & b_{ \pm} \\
0 & d_{ \pm} & 0 \\
0 & a_{ \pm} & 0
\end{array}\right], \quad c_{0}=\left[\begin{array}{ccc}
1 & 0 & 0 \\
0 & 1 & 0 \\
0 & 0 & 0
\end{array}\right] .
$$

In the case when all four of the coefficients $a_{ \pm}, b_{ \pm}$are different from zero, an $A P_{W}$ factorization exists and can be explicitly constructed (see Appendix $\mathrm{E}$ in the supplement). Due to the commutativity condition (4.7), the number of non-zero entries among $a_{ \pm}, b_{ \pm}$cannot equal one. However, there remain cases of exactly two or three non-zero numbers $a_{ \pm}, b_{ \pm}$, and in these cases the $A P$ factorability of the corresponding matrices $G$ is still unknown.

\section{REFERENCES}

[1] M. Bakonyi, L. Rodman, I. Spitkovsky, and H. Woerdeman, Positive extensions of matrix functions of two variables with support in an infinite band, C. R. Acad. Sci. Paris Sér. I Math. 323 (1996), no. 8, 859-863. MR 97i:47023

[2] M. A. Bastos, Yu. I. Karlovich, I. M. Spitkovsky, and P. M. Tishin, On a new algorithm for almost periodic factorization, Operator Theory: Advances and Applications 103 (1998), 53-74. CMP 98:16

[3] C. Corduneanu, Almost periodic functions, J. Wiley \& Sons, 1968. MR 58:2006

[4] N. K. Karapetjanc and S. G. Samko, The functional equation $\psi(x+\alpha)-b(x) \psi(x)=g(x)$, Izv. Akad. Nauk Armjan. SSR. Ser. Mat. 5 (1970), no. 5, 441-448. MR 44:2101

[5] Yu. I. Karlovich, On the Haseman problem, Demonstratio Math. 26 (1993), 581-595. MR 95a:47048

[6] Yu. I. Karlovich and I. M. Spitkovsky, Factorization of almost periodic matrix-valued functions and the Noether theory for certain classes of equations of convolution type, Mathematics of the USSR, Izvestiya 34 (1990), 281-316. MR 90f:47034

[7] _ (Semi)-Fredholmness of convolution operators on the spaces of Bessel potentials, Operator Theory: Advances and Applications 71 (1994), 122-152. MR 95h:47034

[8] - Almost periodic factorization: An analogue of Chebotarev's algorithm, Contemporary Math. 189 (1995), 327-352. MR 96h:47024

[9] _ Factorization of almost periodic matrix functions, J. Math. Anal. Appl. 193 (1995), 209-232. MR 96m:47047]

[10] — Semi-Fredholm properties of certain singular integral operators, Operator Theory: Advances and Applications 90 (1996), 264-287. MR 97k:47046

[11] B. M. Levitan, Almost periodic functions, GITTL, Moscow, 1953 (in Russian). [MR 15:700a

[12] B. M. Levitan and V. V. Zhikov, Almost periodic functions and differential equations, Cambridge University Press, 1982. MR 84g:34004

[13] G. S. Litvinchuk and I. M. Spitkovsky, Factorization of measurable matrix functions, Birkhäuser Verlag, Basel and Boston, 1987. MR 90g:47030

[14] Yu. Lyubarskii and I. Spitkovsky, Sampling and interpolating for a lacunary spectrum, Royal Society of Edinburgh, Proceedings 126A (1996), 77-87. MR 97b:41004 
[15] L. Rodman, I. M. Spitkovsky, and H. J. Woerdeman, Carathéodory-Toeplitz and Nehari problems for matrix valued almost periodic functions, Trans. Amer. Math. Soc. 350 (1998), 2185-2227. MR 98h:47023

[16] I. M. Spitkovsky, On the factorization of almost periodic matrix functions, Math. Notes 45 (1989), no. 5-6, 482-488. MR 90k:47033

[17] I. M. Spitkovsky and H. J. Woerdeman, The Carathèodory-Toeplitz problem for almost periodic functions, J. Functional Analysis 115 (1993), no. 2, 281-293. MR 94f:47020

Department of Mathematics, The College of William and Mary, Williamsburg, VA $23187-8795$

E-mail address: ilya@math.wm.edu

Department of Applied Mathematics, University of Washington, Seattle, WA 98195

E-mail address: dyong@u.washington.edu 